\title{
Erratum to: Grapevine colonization by endophytic bacteria shifts secondary metabolism and suggests activation of defense pathways
}

\author{
Sebastiàn Lòpez-Fernàndez • Stéphane Compant • \\ Urska Vrhovsek • Pier Luigi Bianchedi • \\ Angela Sessitsch • Ilaria Pertot • Andrea Campisano
}

Published online: 13 October 2015

(C) Springer International Publishing Switzerland 2015

\section{Erratum to: Plant Soil \\ DOI 10.1007/s11104-015-2631-1}

The authors wish to apologize to the readers and correct the following errors appeared in the paper in question:

\section{Main Text}

Page 1: "procyanidin 1" should be replaced by "procyanidin B1".

Page 9: "PaVv7 (Fig. 6c-d), SpVs6 (Fig. 6e-f) or control treatment (Fig. 6 g-h)" should be replaced by "PaVv7 (Fig. 6c-e) or control treatment (Fig. 6f-g)".

Page 9: "ca.27\% are organic (hydroxycinnamic) acids" should be replaced by "ca. $27 \%$ are cinnamic (hydroxycinnamic) acids".

The online version of the original article can be found at http://dx. doi.org/10.1007/s11104-015-2631-1

S. Lòpez-Fernàndez $(\bowtie) \cdot$ U. Vrhovsek $\cdot$ P. L. Bianchedi •

I. Pertot $\cdot$ A. Campisano

Research and Innovation Center, Sustainable Agroecosystems and Bioresources Department, Fondazione Edmund Mach,

Via Mach 1, 38010 San Michele all'Adige, TN, Italy

e-mail: juan.lopezfernandez@fmach.it

S. Compant $\cdot$ A. Sessitsch

Health \& Environment Department, AIT Austrian Institute of Technology GmbH, Konrad-Lorenz-Straße 24, 3430 Tulln, Austria

\section{Legends}

Legend Fig. 2 g "exodermis, cortex and endodermis" should be replaced by "rhizodermis, exodermis and cortex".

Legends Figs. 2 and 3 "tracheids" should be replaced by "xylem vessels".

Fig. 3 legend "in Vitis vinifera $\mathrm{L}$. (d-l)" should be replaced by "in $V$. vinifera $\mathrm{L}$. $(\mathbf{d}-\mathbf{q})$ ".

Legend Fig. 5: "done on a root tip" should be replaced by "done on a main root".

Legend Fig. 6: "f strain SpVs6 colonizing main root of Vitis vinifera" should be replaced by "f-g control plants".

Legend Fig. 8: "(green plot)" should be replaced by "(yellow plot $)$ ", and "(yellow plot $)$ " should be replaced by "(green plot)".

Legend Fig. 9: "(green plot)" should be replaced by "(blue plot $)$ ", and "(yellow plot $)$ " should be replaced by "(red plot $)$ ". 